\title{
Muerte súbita por alotrofagia y hemoncosis en una cabra (Capra aegagrus hircus) del departamento de córdoba, Colombia
}

\section{Sudden death by allotriophagy and Haemonchosis in a goat (Capra aegagrus hircus) of Córdoba department, Colombia}

\author{
Cardona A José ${ }^{*}$ Ph.D; Álvarez P Jaime ${ }^{2}$ M.Sc; Pérez O Jair ${ }^{3}$ Ph.D. \\ ${ }^{1}$ Universidad de Córdoba, Facultad de Medicina Veterinaria y Zootecnia, Departamento de Ciencias Pecuarias. \\ Grupo de Investigación en Medicina de Grandes Animales (MEGA), Montería, Colombia. \\ 2Universidad de Córdoba, Facultad de Medicina Veterinaria y Zootecnia, \\ Departamento de Ciencias Pecuarias. Montería, Colombia. \\ ${ }^{3}$ Universidad de La Salle, Facultad de Ciencias Agropecuarias, Bogotá, Colombia.
}

Keywords:

Pica;

plasticphagy;

depraved appetite;

Haemonchus contortus.

\section{Palabras Clave:}

Pica;

plasticofagia;

depravación del gusto;

Haemonchus contortus.
INFORMACIÓN

Recibido: 05-02-2017;

Aceptado: 10-06-2017.

Correspondencia autor:

cardonalvarez@hotmail.com

\begin{abstract}
We report the case of a domestic goat of the Alpine race, whole male, about 6 years old, which was attended by the animal pathology service of the Faculty of Veterinary Medicine and Animal Science at the University of Cordoba. The anamnesis indicates that the animal was found dead in the sheepfold in the morning, with no reports of traumas or illness on the part of the operator, so he was transferred to the necropsy room of the area of animal pathology. The external examination of the corpse showed: poor body condition, hirsute coat, pale mucosa and marked abdominal distention. Necropsy revealed anemia (pallor in the ocular and buccal mucosa, as well as in muscles and in the shortage of subcutaneous adipose tissue), hydrothorax and hydroperitoneum were found, pre-stomachs were enlarged with the presence of gas and solid material. When the rumen was dissected, large quantities of plastic bags were found, as well as a large quantity of mango seeds (Mangifera spp). When the abomasum was dissected, the erythematous and edematized abomasal mucosa was detected in the presence of large numbers of red parasites Haemonchus contortus type. Adult parasites and fecal material were collected from the rectal ampulla and sent to the parasitology laboratory of The Faculty of Veterinary Medicine and Animal Science at the University of Córdoba for classification. The definitive diagnoses of the cadaver were marked anemia, parasitic abomasitis caused by Hemoncosis (Haemonchus contortus), ruminal overload by allotrophagy (plasticphagy), these being the probable causes of death of the animal.
\end{abstract}

\section{Resumen}

Se expone el caso de una cabra doméstica de raza alpina, macho entero, de 6 años de edad aproximadamente, la cual fue atendida por el servicio de patología animal de la Facultad de Medicina Veterinaria y Zootecnia de la Universidad de Córdoba. La anamnesis indica que el animal fue encontrado muerto en el aprisco en horas de la mañana, sin reportes de antecedentes de traumas o enfermedad por parte del operario, por lo que fue trasladado a la sala de necropsia del área de patología animal. Al examen externo del cadáver se observó: pobre condición corporal, pelaje hirsuto, mucosas pálidas y marcada distención abdominal. La necropsia evidenció anemia (palidez en las mucosas ocular y bucal, así como en los músculos y en la escasez de tejido adiposo subcutáneo), se encontró hidrotórax e hidroperitoneo, los pre-estómagos se encontraron aumentados de tamaño con presencia de gas y material sólido. Al diseccionar el rumen se hallaron grandes cantidades de bolsas plásticas, así como gran cantidad de semillas de mango (Mangifera spp). Al diseccionar el abomaso, se detectó la mucosa abomasal eritematosa y edematizada con presencia de grandes cantidades de parásitos 
redondos de color rojo tipo Haemonchus contortus, por lo que fueron colectados parásitos adultos y materia fecal de la ampolla rectal y enviados al laboratorio de parasitología de la Facultad de Medicina Veterinaria y Zootecnia de la Universidad de Córdoba para su clasificación. Los diagnósticos definitivos del cadáver fueron: anemia marcada, abomasitis parasitaria por Hemoncosis (Haemonchus contortus), sobrecarga ruminal por alotrofagia (plasticofagia), siendo éstas las causas probables de la muerte del animal.

\section{Introducción}

La alotrofagia, se considera como la aberración o perversión del apetito, que en la mayoría de los casos es persistente y en ocasiones compulsiva; caracterizado por un desorden en la ingesta de alimentos que usualmente son materiales no comestibles, siendo las más comunes la plasticofagia (plástico), litofagia (piedras), osteofagia (huesos), geofagia (tierra), tricofagia (pelos) y lignofagia (madera) (MARTINS et al., 2004; FIESTAS-TEQUE, 2014; ROSSANIGO et al., 2014). Este trastorno también recibe el nombre de pica, malacia, pseudorexia o parorexia (CAMPUZANO, 2011).

La deficiencia de macro ( $\mathrm{Ca}, \mathrm{P}$ y $\mathrm{Mg}$ ) y micro minerales (Fe, Cu, Mn, Se, Zn y Co), es considerada como la principal causa de alotrofagia en rumiantes; especialmente la deficiencia de fosforo (P) (OCAL et al., 2008).

Se ha informado presentación de pica tanto en animales jóvenes como adultos, sin embargo, en los jóvenes se asocia con la presencia de tricobezoares (masas de pelo ingerido mezclado con alimento y fibra), quizás por el hábito de lamerse el sudor unos a otros como intento de suplir este requerimiento que probablemente sea deficiente en sodio ( $\mathrm{Na}$ ), mientras que en los adultos es más común encontrar la presencia de plásticos, piedras, huesos, fragmentos de vidrio, objetos metálicos como clavos y alambres (MARTINS et al., 2004; FIRYAL, 2007; OCAL et al., 2008; SIMSEK et al., 2015).

Los cuerpos extraños son retenidos en el rumen o retículo, pudiendo quedar mucho tiempo, mientras que los materiales puntiaguda (clavos o vidrios) pueden atravesar la pared del retículo o rumen y llegar al pericardio o peritoneo (retículopericarditis o reticuloperitonitis) pudiendo ocasionar la muerte del animal (MARTINS et al., 2004).

El diagnostico de cuerpo extraños se hace teniendo en cuenta los antecedentes de pica o alotrofagia, sumado a presencia de uno o más signos clínicos característicos de deficiencia de minerales (mala condición corporal, pelaje hirsuto, anemia, distensión abdominal) y hallazgos en la laparotomía y ruminotomia exploratoria (MARTINS et al., 2004; GONZÁLEZ y VALENCIA, 2011).

La Hemoncosis es una parasitosis causada por helmintos de la clase Nematoda, pertenecientes a la familia
Trichostrongylidae, llamado Haemonchus contortus, también conocido como "gusano rojo del cuajar", miden entre 10 y $30 \mathrm{~mm}$ y se localiza en la mucosa del abomaso; por ser hematófago, es considerado uno de los parásitos más patógenos ya que puede consumir $0,08 \mathrm{ml}$ de sangre por día, lo que conlleva a graves estados de anemia, hipoalbuminemia, pérdida de peso y ocasionalmente diarrea (AMADUCl et al., 2016; ZÁRATE et al., 2017).

El objetivo de este estudio fue analizar y describir un caso de muerte súbita por alotrofagia y hemoncosis de una cabra del departamento de Córdoba (Colombia).

\section{Materiales y métodos}

Una cabra doméstica de raza alpina, macho entero, de 6 años de edad aproximadamente, fue atendida por el servicio de patología de la Facultad de Medicina Veterinaria y Zootecnia de la Universidad de Córdoba.

Entre los datos anamnesicos, se tiene que el animal fue encontrado muerto en el aprisco en horas de la mañana, no se reportaron antecedentes de enfermedad por parte del operario, por lo que se trasladó al área de patología donde se le realizó la necropsia.

Al examen externo del cadáver se observó pelaje hirsuto, mucosas pálidas y marcada distención abdominal. En la necropsia, se encontraron signos importantes como: anemia (palidez en las mucosas ocular y bucal) (Figura 1), palidez de los músculos, escasez de tejido adiposo subcutáneo, hidrotórax e hidroperitoneo. Los pre-estómagos se encontraron aumentados de tamaño, y al diseccionar el rumen fue evidenciado gran cantidad de bolsas plásticas en su interior, así como gran cantidad de semillas de mango (Mangifera spp) (Figura 2). Al diseccionar el abomaso, se detectó la mucosa abomasal eritematosa y edematizada con presencia de grandes cantidades de parásitos redondos de color rojo (Figura 3 ), por lo que fueron colectados parásitos adultos y materia fecal de la ampolla rectal y enviados para su clasificación al laboratorio de parasitología de la Facultad de Medicina Veterinaria y Zootecnia de la Universidad de Córdoba, donde fueron cuantificados mediante la técnica de McMaster, grandes cantidades de huevos de parásitos (>1000 HPG) y clasificados como Haemonchus contortus (Figura 4). 


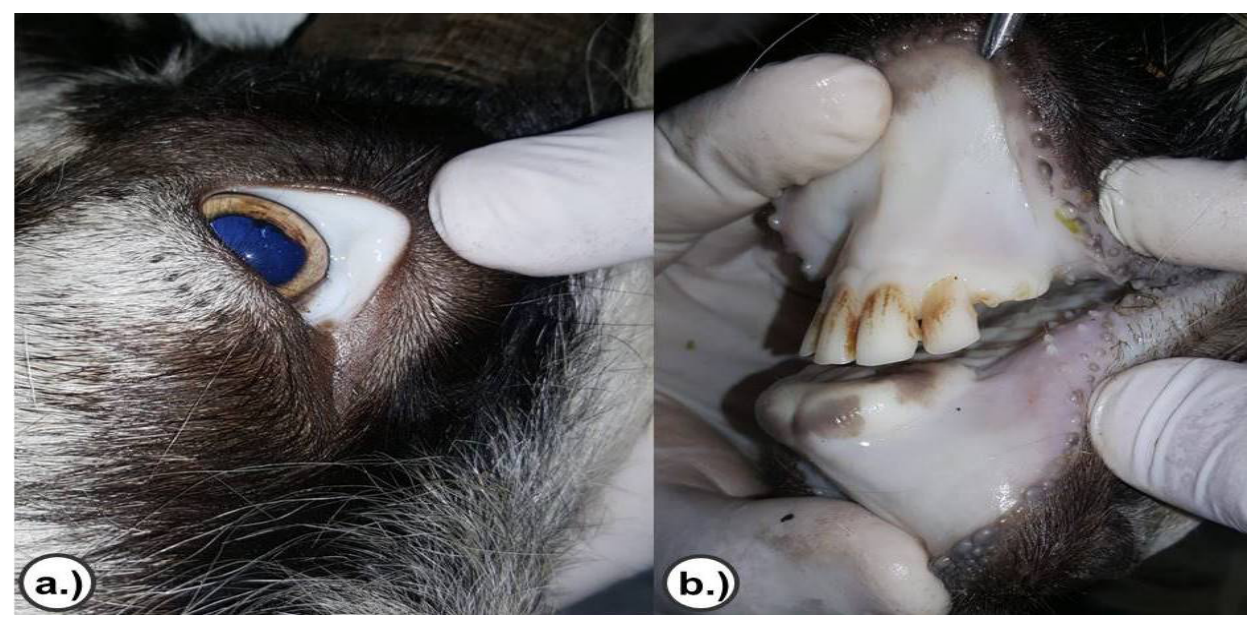

Figura 1. Marcada anemia grado 5 en escala FAMACHA. Observe el color pálido en las mucosas a.) ocular y b.) bucal.

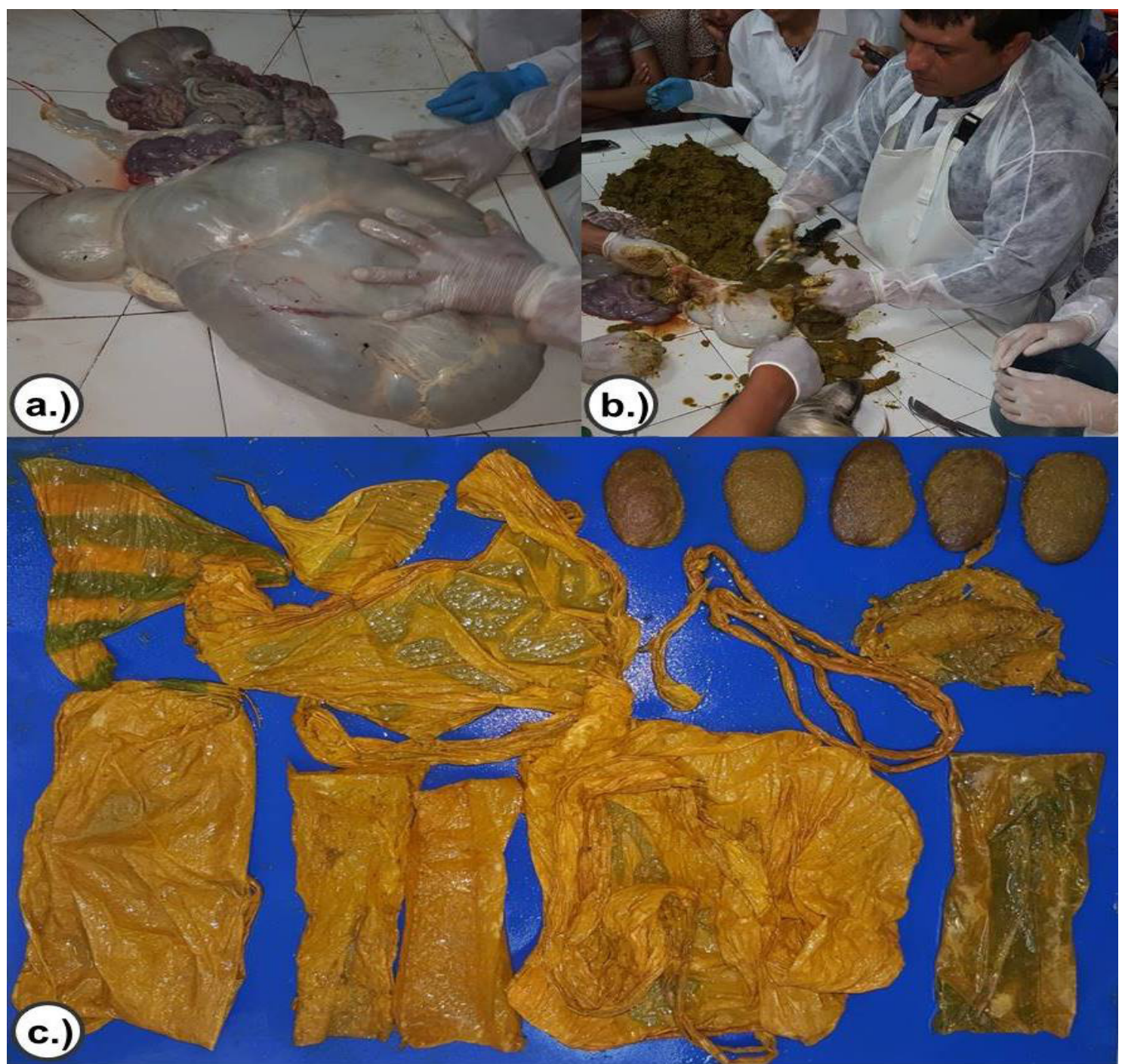

Figura 2. Procedimiento de necropsia. a.) Pre-estómagos con aumento considerable de tamaño. b.) Proceso de disección de rumen. c.) Grandes cantidades de bolsas de plástico y semillas de mango (Mangifera spp). 


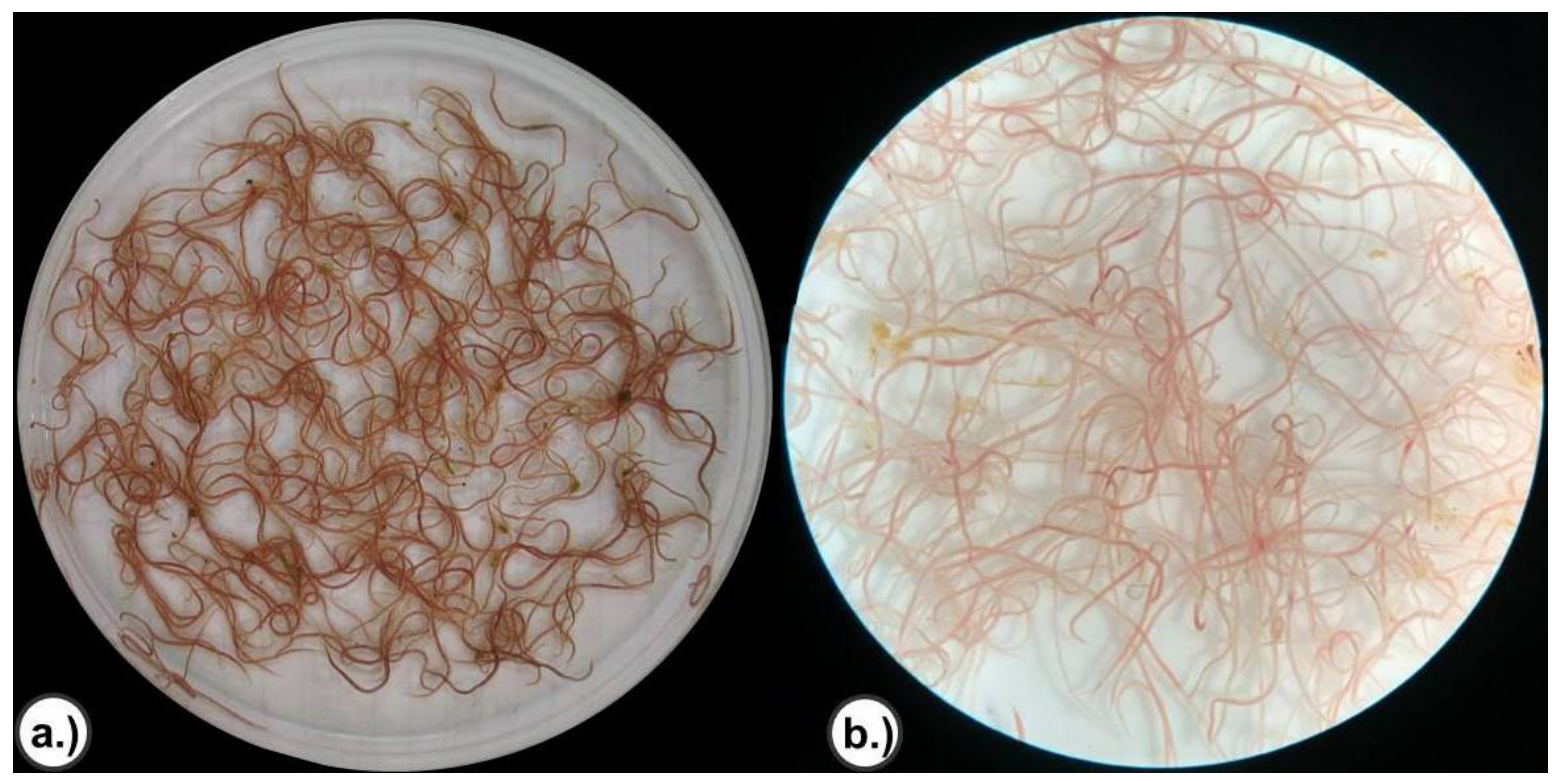

Figura 3. Estados adultos de Haemonchus contortus. Observe las grandes cantidades de parásitos redondos hallados en el abomaso. a.) en caja de Petri. b.) Observados en estereoscopio.

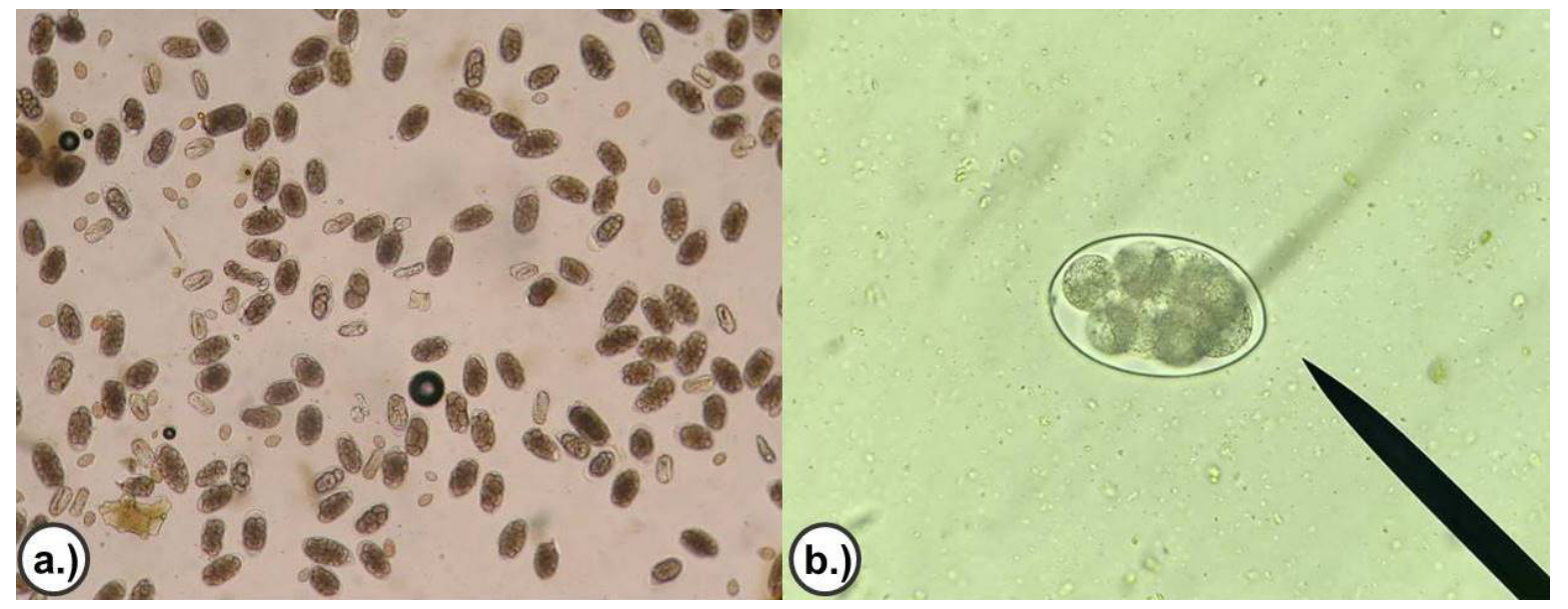

Figura 4. Huevos de Haemonchus contortus observados en el microscopio. a.) a 10x y b.) a 20x.

\section{Resultado y discusión}

No existen reportes en la literatura consultada sobre muerte súbita por alotrofagia en cabras del departamento de Córdoba y Colombia.

La anemia marcada que presentó el cadáver, se correlaciona con la cuantificación de huevos por gramo de la muestra coprológica (>1000 HPG) y con la cantidad de parásitos adultos encontrados en el abomaso, caracterizándose en el grado 5 en la escala de FAMACHA, ya que según BESIER et al. (2016), existe una estrecha relación entre el nivel de parasitismo gastrointestinal y el grado de anemia según el color de la mucosa ocular en la escala FAMACHA.

Las manifestaciones de alotrofagia encontradas en el cadáver (anemia, pérdida de peso, pelaje hirsuto, plasticofagia), coinciden con lo reportado por OCAL et al. (2008); OLIVEIRA et al. (2010) y LOPES et al. (2013), quienes manifiestan que en las deficiencias de minerales se presentan signos inespecíficos como, despigmentación del pelo y piel, pérdida de peso, mucosas pálidas, muerte súbita, anemia, hirsutismo, sobrecarga ruminal y pica. 
La muerte súbita de la cabra del presente estudio se relaciona con la plasticofagia, concordando con MARTINS et al. (2004); GONZÁLEZ y VALENCIA, (2011) y SIMSEK et al. (2015), quienes expresan que la ingesta de bolsas de plástico o fragmentos de este, comúnmente ocasiona obstrucciones del píloro, seguido de una distención abdominal a nivel de la fosa del ijar izquierda, por inhibición del reflejo del eructo, acumulación de gas y consecuente timpanismo, de igual forma, el acumulo de materiales pequeños y compactos como pequeñas piedras, grava y arena en rumen, predispondrán a impacciones, en los que se evidencia una distención abdominal en la zona ventral izquierda.
La edad del animal fue de 6 años aproximadamente, siendo la alotrofagia relacionada con la etapa productiva, es así como SIMSEK et al. (2015), expresan que hallazgo de plásticos, huesos y piedras son más comunes en animales mayores.

Los diagnósticos definitivos del cadáver fueron: anemia marcada, abomasitis parasitaria por Hemoncosis (Haemonchus contortus), sobrecarga ruminal por alotrofagia (plasticofagia), siendo éstas las causas probables de la muerte del animal, de acuerdo a lo descrito por LOPES et al. (2013); SIMSEK et al. (2015); AMADUCl et al. (2016) y ZÁRATE et al. (2017).

\section{Referencias}

AMADUCI, A.; BORGES, J.; SITKO, M.; MARTINES, T.; SILVA, J., SANTOS, A.; FERREIRA, G.; ALMADA, A.; PIAU, R. 2016. Parâmetros sanguíneos e OPG (ovos por grama de fezes) de ovelhas mestiças da raça Dorper em diferentes graus do método Famacha. Arq. Ciênc. Vet. Zool. 19 (4): 221-225.

BESIER, R.; KAHN, L.; SARGISON, N.; VAN WYK, J. 2016. Diagnosis, treatment and management of Haemonchus contortus in small ruminants. Adv Parasitol 93: 181-238.

CAMPUZANO, G. 2011. Pica: el síntoma olvidado. Medicina \& Laboratorio. 17(11-12):533-552.

FIESTAS-TEQUE, L. 2014. Pica en anemia severa: a propósito de un caso. Rev. Neuro-Psiquiatría. 77(2):123-127.

FIRYAL, S. 2007. Pica (Depraved appetite; allotrophagia) in domestic animals. Pakistan Vet. J. 27 (4): $208-210$.

GONZÁLEZ, S.; VALENCIA, A. 2011. Reporte de Caso: Indigestion Ruminal en un Bovino por elementos Indigestibles. Rev. Fac. Cienc. Agr. 3(1): 47-53.

LOPES, A.; MACHADO, R.; DE TARSO LANDGRAF, P. 2013. Deficiência de cobre condicionada a altos teores de zinco, manganês e ferro na região do Médio Paraíba, RJ, Brasil. Semina: Ciênc. Ag. 34 (3): 1293-1300.

MARTINS, A.; LEME, M.; PORTUGAL, M.; BALDASSI, L.; MARGATHO, L. 2004. Presença de corpos estranhos no habituais no aparelho digestório dos bovinos. Arq. Inst. Biol. 71(1):83-87.

OCAL, N.; GOKCE, G.; GUCU, A.; UZLU, E.; YAGCI, B.; URAL, K. 2008. Pica as a predisposing factor for traumatic reticuloperitonitis in dairy cattle: serum mineral concentrations and hematological findings. J. Anim. Vet. Advan. 7(6):651-656.

OLIVEIRA, C.; DE MATTOS F. 2010. Funções e sintomas de deficiência dos minerais essenciais utilizados para suplementação dos bovinos de corte. UNICiências. 14(2): 199-223.

ROSSANIGO, C.; FRASINELLI, C.; BENGOLEA, A. 2014. Pica en vacas en parición confinadas con silo de autoconsumo y agua de bebida de bajo tenor salino. Rev. Arg. Prod. Anim. 34(Supl 1):45-75.

SIMSEK, A.; SEKIN, S.; ICEN, H.; KOCHAN, A.; CELIK, O.; YAMAN, T. 2015. Abomasal impaction due to sand accumulation in two cows. Large An. Rev. 21(3):125-127.

ZÁRATE, D.; ROJAS, J.; SEGURA, A. 2017. Validación del Método FAMACHA@ para dosificación antihelmíntica selectiva en rebaños caprinos lecheros. Rev Inv Vet Perú. 28(1): 150-159 